\title{
Evidence that Brain-Derived Neurotrophic Factor from Presynaptic Nerve Terminals Regulates the Phenotype of Calbindin-Containing Neurons in the Lateral Septum
}

\author{
James P. Fawcett, Mario A. Alonso-Vanegas, Stephen J. Morris, Freda D. Miller, Abbas F. Sadikot, and \\ Richard A. Murphy \\ Center for Neuronal Survival and Division of Neurosurgery, and Montreal Neurological Institute and the Department of \\ Neurology and Neurosurgery, McGill University, Montreal, Quebec, Canada H3A 2B4
}

\begin{abstract}
Brain-derived neurotrophic factor (BDNF) is transported anterogradely in neurons of the CNS and can be released by activitydependent mechanisms to regulate synaptic plasticity. However, few neural networks have been identified in which the production, transport, and effects of BDNF on postsynaptic neurons can be analyzed in detail. In this study, we have identified such a network. BDNF has been colocalized by immunocytochemistry with tyrosine hydroxylase $(\mathrm{TH})$ in nerve fibers and nerve terminals within the lateral septum of rats. BDNF-containing nerve fibers terminate on a population of calbindin-containing neurons in lateral septum that contain TrkB, the high-affinity receptor for BDNF. Overexpression of BDNF in noradrenergic neurons increased levels of calbindin in septum, as well as in whole-brain lysates. Septal levels of calbindin and BDNF partially decreased after unilateral lesions
\end{abstract}

of the medial forebrain bundle (MFB), induced with 6-hydroxydopamine, a treatment that abolished TH staining. These data suggest that BDNF is anterogradely transported within the MFB in catecholaminergic neurons arising from brainstem nuclei. To determine whether BDNF affects the production of calbindin in lateral septal neurons directly, we tested the effects of BDNF on cultures of septal neurons from embryonic day 16-17 rats. BDNF promoted the expression of calbindin, as well as the arborization of calbindin-containing neurons, but BDNF had no effect on cell division or survival. Together, these results suggest that BDNF, anterogradely transported in catecholaminergic neurons, regulates calbindin expression within the lateral septum.

Key words: BDNF; calbindin; catecholaminergic neurons; septum; anterograde transport; TrkB
Brain-derived neurotrophic factor (BDNF) produced in brain neurons promotes neuronal survival, differentiation (for review, see Snider, 1994), and synaptic plasticity (for review, see Thoenen, 1995; McAllister et al., 1999). Neurons release BDNF by activity-dependent mechanisms (Goodman et al., 1996; Aloyz et al., 1999; Mowla et al., 1999), which is consistent with BDNF being sorted into the regulated secretory pathway of neurons (Mowla et al., 1999) and anterogradely transported in axons (von Bartheld et al., 1996; Altar et al., 1997; Fawcett et al., 1998) (for review, see Altar and DiStephano, 1998).

The lateral septum, which is involved in limbic activity, including learning and memory (Urban et al., 1995; Jaffard et al., 1996), contains high levels of BDNF protein (Conner et al., 1997) but little or no BDNF mRNA (Castren et al., 1995; Conner et al., 1997), suggesting that BDNF is transported into the lateral septum via afferent nerve fibers arising from neurons elsewhere in the brain. Brainstem and specifically midbrain catecholaminergic neurons are candidate sources for providing BDNF to the septum. BDNF is present in axons and terminals of neurons con-

Received July 19, 1999; revised Oct. 5, 1999; accepted Oct. 20, 1999.

This work was funded by a Program Grant (F.D.M. and R.A.M.) and operating grants (A.F.S.) from the Medical Research Council of Canada and The March of Dimes. J.P.F. was funded by a Rick Hansen studentship, M.A.V. is a Jeanne Timmins Fellow of the Montreal Neurological Institute (MNI), A.F.S. is a Medical Research Council Scholar, and F.D.M. is an MNI Killam Scholar. We thank Irene Mazzoni, Jacynthe LaLiberté, and Marie Claude Bélanger for their assistance.

Correspondence should be addressed to Dr. Richard A. Murphy, Montreal Neurological Institute, McGill University, 3801 University Avenue, Montreal, Quebec, Canada H3A 2B4. E-mail: director@mni.lan.mcgill.ca.

Copyright @ 1999 Society for Neuroscience 0270-6474/99/200274-09\$15.00/0 taining dopamine- $\beta$-hydroxylase (Fawcett et al., 1998). Catecholaminergic neurons in the locus ceruleus (LC), ventral tegmental area (VTA), medial substantia nigra pars compacta (SNPC), and hypothalamus all produce BDNF mRNA (Seroogy and Gall, 1993; Venero et al., 1994; Conner et al., 1997). Axons from these neurons travel via the medial forebrain bundle (MFB) into the lateral septum (Swanson, 1982; Jakab and Leranth, 1995). Furthermore, almost $90 \%$ of neurons in the ventral tegmental nucleus containing mRNA coding for BDNF also express tyrosine hydroxylase (TH) (Seroogy and Gall, 1993).

Within the lateral septum, catecholamine-containing axons form pericellular baskets surrounding GABAergic spiny neurons, most of which express calbindin (Jakab and Leranth, 1990, 1995; Seifert et al., 1998), a member of the EF handed protein family that buffer intracellular $\mathrm{Ca}^{2+}$ (Baimbridge et al., 1992). BDNF induces calbindin production in neurons cultured from hippocampus (Ip et al., 1993) striatum (Ventimiglia et al., 1995), cortex (Pappas and Parnavelas, 1997), and cerebellum (Larkfors et al., 1996). Furthermore, BDNF increases the size and arborization of calbindin-containing neurons (Ventimiglia et al., 1995; Pappas and Parnavelas, 1997), and BDNF null mutant mice show reduced levels of total brain calbindin (Jones et al., 1994). Therefore, BDNF produced within catecholaminergic neurons may have a role in regulating the phenotype of calbindin-containing neurons.

Results in this study confirm that prediction. Data show that BDNF-containing catecholaminergic neurons traveling via the MFB terminate on calbindin-containing neurons in the lateral septum that contain TrkB. Overexpressing BDNF in noradren- 
ergic axons increases calbindin levels in the septum. Conversely, disrupting the MFB with 6-hydroxydopamine (6-OHDA) reduces septal levels of calbindin, as well as TH and BDNF. BDNF promotes calbindin synthesis in cultured septal neurons, as well as the branching of calbindin-containing neurons. BDNF had no effect on cell proliferation or cell death. Thus, BDNF in MFB fibers terminating within the lateral septum may provide a useful model system for studying the physiology of BDNF, particularly its production, transport, and effects on postsynaptic neurons.

\section{MATERIALS AND METHODS}

Antibodies and reagents. For immunocytochemistry, we used rabbit antibodies to BDNF (anti-BDNF), kindly supplied by Drs. Qia Yan and Andrew Welcher at Amgen (Thousand Oaks, CA) (Yan et al., 1997). For Western blotting, anti-BDNF was obtained from Santa Cruz Biotechnology (Santa Cruz, CA). Antibodies raised in rabbit to calbindin were obtained from Swant (Bellizona, Switzerland), and mouse antibodies to calbindin were obtained from Sigma (St. Louis, MO). Anti-tyrosine hydroxylase was obtained from Incstar (Stillwater, MN). Dr. Stewart Feinstein (Santa Barbara, CA) kindly supplied rabbit antibody to TrkB. 5-Bromo-2'-deoxyuridine (BrdU) reagent was purchased from Boehringer Mannheim (Quebec, Canada). Secondary antibodies for immunocytochemistry were purchased from Jackson ImmunoResearch (Ontario, Canada) and for Western blotting from Boehringer Mannheim. ECL reagent was purchased from NEN Life Science Products (Mandel Scientific, Quebec, Canada). All other chemicals, unless otherwise noted, were purchased from Sigma.

Animals. Adult and timed pregnant rats were obtained from Charles River (Montreal, Canada) and were handled according to the protocols described by the Canadian Council on Animal Care.

Lesions. Female Sprague Dawley rats (Charles River) weighing between 180 and 200 gm were deeply anesthetized by intraperitoneal injection using a mixture of ketamine (Ketaset, Ayerst; $90 \mathrm{mg} / \mathrm{ml}$ ) and xylazine (Rompun, Haven; $10 \mathrm{mg} / \mathrm{ml}$ ). 6-Hydroxydopamine-hydrobromide (Research Biochemicals, Natick, MA) in $5 \mu$ l of $0.9 \%$ saline containing $0.2 \mathrm{mg} / \mathrm{ml}$ ascorbate was injected into the right medial forebrain bundle at the following coordinates: toothbar, -2.4 ; anterior, -4.4 ; lateral, 1.0; ventral, 7.5. Two weeks after the lesion, animals were tested for rotational behavior in automated rotormeter bowls (San Diego Instruments, San Diego, CA) after injection of amphetamine (5 mg/kg, i.p.).

Cell culture. Methods for culturing septal neurons were similar to those used by Mazonni and Kenisberg (1991). Briefly, the septal region of the brain was removed from embryonic rat pups [embryonic day (E) 16 to E17], incubated for $20 \mathrm{~min}$ at $37^{\circ} \mathrm{C}$ in $0.2 \%$ trypsin, dissociated in a pipette, and plated at a density of $3.5 \times 10^{5}$ cells $/ \mathrm{cm}^{2}$ in tissue culture dishes coated with $(10 \mu \mathrm{g} / \mathrm{ml})$ poly-L-lysine. Cells were cultured in Neurobasal media containing $500 \mu \mathrm{M}$ glutamine and $25 \mu \mathrm{M}$ glutamate with B-27 and penicillin-streptomycin supplements. Two days after plating, $50 \mathrm{ng} / \mathrm{ml}$ human recombinant BDNF (Regeneron Pharmaceuticals, Tarrytown, NY) was added to cultures. For cell feeding, $50 \%$ of the old medium was removed and the remainder supplemented with fresh medium without glutamate, with or without fresh neurotrophin. Five days after BDNF was first added to media, the cells were processed for immunocytochemistry (see below) or for Western blotting. For Western blot analysis, cells were washed three times in $37^{\circ} \mathrm{C} \mathrm{PBS}$ and exposed for $20 \mathrm{~min}$ to ice-cold Tris lysis buffer containing $137 \mathrm{~mm} \mathrm{NaCl}, 20 \mathrm{~mm}$ Tris, $\mathrm{pH} 8.0,1 \%$ (v/v) NP-40, 10\% (v/v) glycerol, $1 \mathrm{mM}$ PMSF, $10 \mu \mathrm{g}$ of aprotinin, and $0.2 \mu \mathrm{g}$ of leupeptin. The lysate was centrifuged at 15,000 rpm for $15 \mathrm{~min}$, and protein concentrations in the supernatant were measured using the BCA kit from Pierce (Rockford, IL).

Dopamine- $\beta$-hydroxylase BDNF mice. Transgenic mice engineered to overexpress BDNF in noradrenergic neurons using the dopamine- $\beta$ hydroxylase $(\mathrm{DBH})$ promoter have been described previously (Causing et al., 1997; Fawcett et al., 1998). Brains from transgenic and control mice were rapidly removed after decapitation and lysed in Tris lysis buffer (described above) (1:10 w/v). Brain lysate was generated as described previously (Fawcett et al., 1997, 1998). Individual septa from the brains of control and transgenic mice were rapidly dissected onto a glass plate kept at $4^{\circ} \mathrm{C}$. Septa were lysed as described above.

Western blotting. Methods were identical to those described previously by Fawcett et al. (1997). Briefly, equal amounts of total protein were electrophoresed by SDS-PAGE, transferred to $0.2 \mathrm{~mm}$ nitrocellulose (Bio-Rad, Hercules, CA), and blocked for $1 \mathrm{hr}$ in $5 \%$ milk in Tris- buffered saline-Triton X-100 (TBST) at room temperature. Primary antibodies were used at dilutions of 1:5000 (BDNF), 1:10000 (tubulin), and 1:5000 (calbindin). After incubation overnight at $4^{\circ} \mathrm{C}$, the blots were washed three times in TBST, incubated in secondary HRP-conjugated goat anti-rabbit or goat anti-mouse antibody for $1.5 \mathrm{hr}$, washed in TBST, and developed using standard ECL protocols, according to the manufacturer's instructions.

Western blot replicas were scanned using a flatbed scanner (Umax, Astra 600s) at 300 dots per inch and saved into Adobe Photoshop 4.0 (Adobe Systems, San Jose, CA). Scanned images were opened in NIH Image (version V1.61; Wayne Rosband, National Institutes of Health, Rockville, MD). Areas of interest were defined, and mean pixel densities were determined. For each of three animals analyzed, the density of calbindin normalized to tubulin on the unlesioned side was used as the control value and compared with the lesioned side for the same animal. Statistical significance was determined using Student's $t$ test for pairwise comparison.

Immunocytochemistry of cultured cells. After $5 \mathrm{~d}$ with or without BDNF, cultures of septal neurons were rinsed three times with $0.1 \mathrm{M}$ phosphate buffer (PB), pH 7.4, and fixed in $4 \%$ paraformaldehyde in $\mathrm{PB}$ for $45 \mathrm{~min}$. The cultures were extensively washed in PBS and blocked for $20 \mathrm{~min}$ in a solution containing $10 \%$ normal goat serum, $0.1 \%$ Triton $\mathrm{X}-100$ in PBS. The cultures were exposed to anti-calbindin (1:5000) overnight at $4^{\circ} \mathrm{C}$, washed three times in PBS, and incubated with $\mathrm{Cy} 2$ conjugated goat anti-mouse antibody, at a final dilution of 1:1000, for 1 hr. Cultures were subsequently washed three times with PBS, and Hoechst 33258 stain was added at 1:5000 dilution for $30 \mathrm{sec}$. The cultures were washed and coverslipped in a $50 \%$ glycerol-PBS solution.

BrdU labeling. Methods for BrdU labeling were similar to those of Pappas and Parnavelas (1997). Briefly, 2 d after plating, cultures of septal neurons were treated for $24 \mathrm{hr}$ with BrdU labeling reagent (1:800), washed extensively in PBS, and replaced with fresh media with or without BDNF $(50 \mathrm{ng} / \mathrm{ml})$. After $3 \mathrm{~d}$, the cultures were rinsed three times in $\mathrm{PB}$, fixed with $4 \%$ paraformaldehyde in $\mathrm{PB}$ for $45 \mathrm{~min}$, washed, and blocked in $10 \%$ normal goat serum and $0.1 \%$ Triton $\mathrm{X}-100$ in PB. Antibody to calbindin (1:1000) was added to the block solution and left overnight at $4^{\circ} \mathrm{C}$. Cultures were washed three times in PBS and incubated for $1 \mathrm{hr}$ at room temperature in $\mathrm{Cy} 3$-conjugated goat anti-rabbit $\mathrm{IgG}$ in PBS. The cultures were rinsed in PBS, followed by incubation for $45 \mathrm{~min}$ in $2 \mathrm{~N} \mathrm{HCL}$ and washing two times for $15 \mathrm{~min}$ each in $0.1 \mathrm{M}$ borate buffer, pH 8.3. After additional washes with PBS, anti-BrdU (Boehringer Mannheim) was added overnight at $4^{\circ} \mathrm{C}$ at a dilution of $1: 10$, as per the manufacturer's recommendation. Cultures were rinsed in PBS, followed by a $1 \mathrm{hr}$ incubation at room temperature in Cy2-conjugated goat anti-mouse in PBS. After two washes in PBS and one $30 \mathrm{sec}$ incubation in Hoechst 33258 (1:5000), cultures were washed a final time in PBS and coverslipped in a glycerol-PBS mixture for fluorescent microscopy.

Fluorescent immunocytochemistry. Methods for identifying BDNF in brain by fluorescence microscopy have been described previously (Fawcett et al., 1998). For colocalizing BDNF and calbindin or calbindin and TrkB, we modified these procedures as follows: rats were killed with sodium pentobarbital $(65 \mathrm{mg} / \mathrm{kg})$, followed by transcardial perfusion using $50 \mathrm{ml}$ of heparanized PBS, followed by $2 \%$ paraformaldehyde in 0.1 $\mathrm{M} \mathrm{PB}, \mathrm{pH}$ 7.3. Brains were removed and post-fixed for $2 \mathrm{hr}$ at $4^{\circ} \mathrm{C}$ and then cryoprotected overnight in $30 \%$ sucrose before processing. All immunostaining was performed on free-floating $12 \mu \mathrm{m}$ sections cut on a Reichart Jung cryostat. After blocking in $10 \%$ normal goat serum and $0.1 \%$ Triton $\mathrm{X}-100$ in $\mathrm{PB}, \mathrm{pH} 7.4$, we added the following primary antibodies: anti-BDNF (rabbit) and anti-calbindin (mouse) at dilutions of 1:2000 and 1:5000, respectively; anti-TrkB (rabbit) and anti-calbindin (mouse) at dilutions of 1:1000 and 1:5000 respectively; and anti-TH (mouse) at a dilution of 1:200. Sections were incubated for a minimum 48 $\mathrm{hr}$ at $4^{\circ} \mathrm{C}$. After three washes in $\mathrm{PB}$ with vigorous shaking, sections were left for $2 \mathrm{hr}$ at room temperature in $\mathrm{Cy} 3$-conjugated goat anti-rabbit (1:1000) mixed with Cy2-conjugated goat anti-mouse (1:000) in the block solution. Sections were then washed in PB three times, mounted on slides, covered with PBS-glycerol, and coverslipped before examination on a Zeiss (Oberkochen, Germany) Axioscope.

Microscopy. Sections for fluorescent microscopy were analyzed on a Zeiss Axioscope system. For epifluorescent microscopy, we used filters designed for double-label analysis of two fluorochromes. Images captured using a CCD model XC-75CE Sony (Tokyo, Japan) camera were saved into Northern Eclipse (version 2.1; Emix Imaging, Inc.) program. Further analysis of images was done using Photoshop 4.0. For confocal microscopy, images were generated on a Zeiss LSM 410 inverted con- 
focal microscope using a $63 \times, 1.4$ NA objective. Images were captured on a photomultiplier after passage through FT590 and LP590 filter sets. Confocal images were captured at a depth of $1 \mu \mathrm{m}$ and imported into Adobe Photoshop 4.0, and false color was added to each filter set. Images were overlaid for colocalization analysis.

Cell counts. For analyzing cell cultures, we captured images of a minimum of 30 random fields of cells for each experimental condition using a $40 \times$ objective and the LCD camera. Data from four different experiments were pooled. Images from the red, green, and blue channels were imported into NIH Image software (version V1.61; Wayne Rosband) for analysis. The number of calbindin-positive, Hoechst-positive, and BrdU-positive cells was separately scored for each field. We also counted the number of neurites emanating from the soma of each calbindin-positive cell. Captured images were imported into Adobe Photoshop 4.0, and individual images were magnified two times. Only those processes extending more than two cell bodies in length were scored as positive.

Statistics. A Student's $t$ test was used where appropriate to compare various treatment groups.

\section{RESULTS}

\section{BDNF and tyrosine hydroxylase colocalize in fibers and nerve terminals in the adult rat lateral septum}

In previous studies, we have shown that BDNF and dopamine$\beta$-hydroxylase colocalize in neurons distributed throughout the brain, consistent with the anterograde transport of endogenous BDNF in the CNS (Fawcett et al., 1998). To determine whether BDNF, which is synthesized in catecholaminergic neurons (Seroogy and Gall, 1993; Castren et al., 1995; Conner et al., 1997), is anterogradely transported in catecholaminergic axons and nerve terminals, we performed double-label immunocytochemical analysis for BDNF and $\mathrm{TH}$, a marker of catecholaminergic axons (Armstrong et al., 1981). We chose to focus on the lateral septum because it is a forebrain area rich in both BDNF- and THcontaining fibers (Lindvall, 1975; Jakab and Leranth, 1990; Conner et al., 1997; Seifert et al., 1998). Furthermore, the lateral septum lacks mRNA coding for BDNF (Castren et al., 1995; Conner et al., 1997) or TH (Cortes et al., 1990), suggesting that both TH and $\mathrm{BDNF}$ are transported into the lateral septum from elsewhere.

Although TH-positive fibers that were not immunoreactive for $\mathrm{BDNF}$ and BDNF fibers that were not immunoreactive for $\mathrm{TH}$ were detected in the lateral septum, many fibers contained both molecules (Fig. 1a,b). These results are consistent with data showing that a subpopulation of midbrain dopaminergic neurons contain BDNF (Seroogy and Gall, 1993). In addition, in the lateral septum, we observed immunoreactivity for both BDNF and $\mathrm{TH}$ in structures (Fig. 1c, $d$, arrowheads) reminiscent of $\mathrm{TH}-$ positive pericellular baskets (Jakab and Leranth, 1995; Seifert et al., 1998). Thus, BDNF is present in the axons and terminals of catecholaminergic neurons, evidence consistent with BDNF being anterogradely transported within these neurons.

\section{BDNF-containing fibers terminate on calbindin-containing neurons}

Because most TH-positive pericellular baskets in the lateral septum surround calbindin-positive GABAergic neurons (Seifert et al., 1998) and because BDNF can alter calbindin expression in neurons grown in vitro (Ip et al., 1993; Ventimiglia et al., 1995; Pappas and Parnavelas, 1997), we questioned whether BDNFcontaining terminals innervate calbindin-containing cells. Double-label immunocytochemistry detected BDNF in punctate terminals that appear to surround some but not all of calbindinpositive cells (Fig. $2 a-c$ ). In agreement with previous results, we never observed calbindin in presynaptic fibers (Seifert et al., 1998). Confocal microscopy revealed BDNF immunoreactivity in
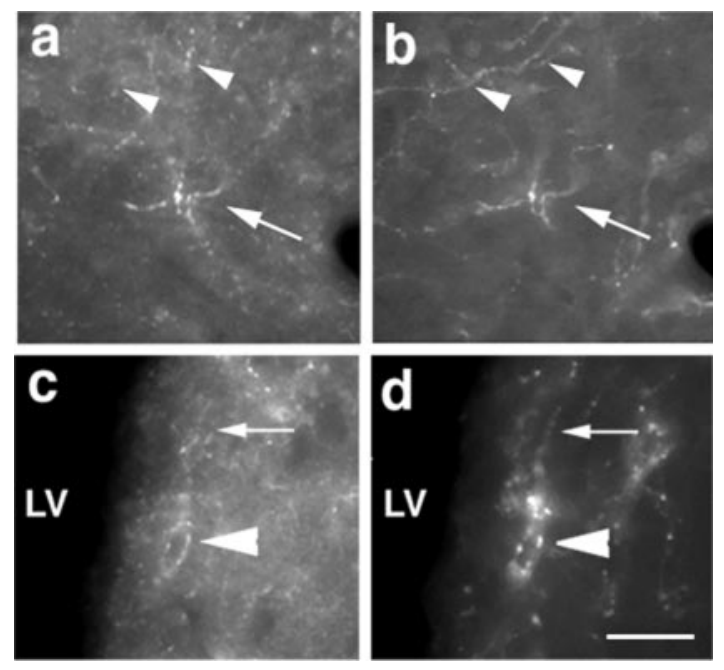

Figure 1. BDNF and TH colocalize in lateral septum. Immunofluorescent micrographs showing immunoreactive BDNF ( $a$ and $c$, arrows) and tyrosine hydroxylase ( $b$ and $d$, arrows) in nerve fibers of lateral septum. Not all TH-containing fibers contain BDNF (compare the arrowheads in $a$ and $b$ ). Immunoreactivity for $\operatorname{BDNF}(c)$ and $\mathrm{TH}(d)$ can also be colocalized in punctate structures forming pericellular basket endings surrounding postsynaptic neurons (arrowheads). Scale bar, $25 \mu \mathrm{m}$.

punctate structures surrounding calbindin-positive neurons (Fig. $2 d$, arrow), although BDNF immunoreactivity was also evident surrounding neurons that did not stain for calbindin (Fig. 2d, arrowhead). Our data showing $\mathrm{TH}$ and BDNF colocalization in pericellular baskets, in addition to BDNF-positive pericellular baskets forming on calbindin-positive neurons, suggest that a population of TH- and BDNF-positive terminals form direct pericellular contacts with calbindin-positive neurons in the lateral septum.

\section{Calbindin-containing septal neurons produce TrkB}

If BDNF released from nerve terminals within lateral septum is to affect calbindin-containing neurons, then calbindin-containing neurons would also be expected to express TrkB, the high-affinity receptor for BDNF. Figure 3 shows that immunoreactivity for $\operatorname{TrkB}(a$, arrow) and calbindin (b, arrow) colocalize in some lateral septum neurons. Figure 3, $a$ and $b$, also shows one neuron (arrowheads) that is TrkB-positive but calbindin-negative. Therefore, there appears to be some neurons in lateral septum that are capable of responding to BDNF that do not contain calbindin.

\section{BDNF regulates calbindin expression in the lateral septum}

Our data indicate that BDNF is anterogradely transported by catecholaminergic afferent nerve fibers and stored presynaptically. They also suggest that BDNF may be secreted from terminals, consistent with reports showing activity-induced release of BDNF results in the rapid activation of TrkB receptors in the CNS (Aloyz et al., 1999). Once released, BDNF may regulate calbindin levels in target neurons. We tested this hypothesis in two ways. First, we used Western blot methods to measure calbindin levels in whole-brain extracts and in the septum of transgenic mice that overexpress BDNF, twofold to fourfold, in noradrenergic neurons under the control of the dopamine- $\beta$-hydroxylase promoter (Causing et al., 1997; Fawcett et al., 1998). Increased levels of calbindin were observed in whole-brain extracts of transgenic animals compared with their littermate controls (Fig. 4a). When reprobed with a rabbit polyclonal antibody raised against a 

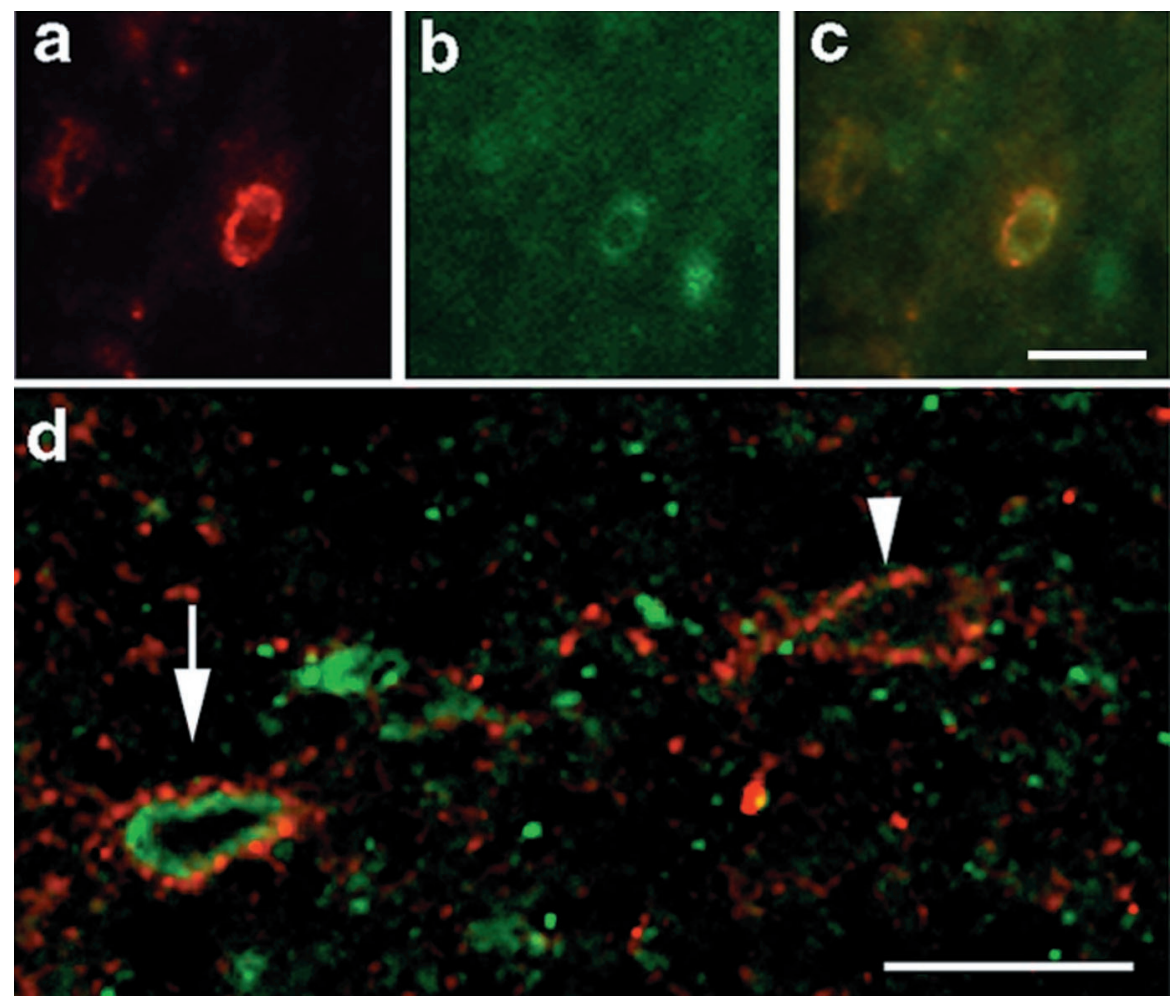

Figure 2. BDNF-containing nerve terminals in lateral septum. $a$, An immunofluorescent micrograph of BDNF immunoreactivity in the lateral septum. Note the punctate appearance of the BDNF immunoreactivity. $b$, The same section as in $a$ showing calbindin immunoreactivity. $c$, Doublelabel fluorescent micrograph of $a$ and $b$ overlaid. Note that the punctate BDNF immunoreactivity (red) surrounds the calbindin immunoreactivity (green). D, Confocal image of BDNF (red) and calbindin (green) immunoreactivity in the lateral septum. The arrow shows punctate BDNF immunoreactivity surrounding a calbindin-positive cell. The arrowhead shows a similar distribution of BDNF immunoreactivity surrounding a presumptive neuron that does not contain immunoreactivity for calbindin. Scale bar, $25 \mu \mathrm{m}$.

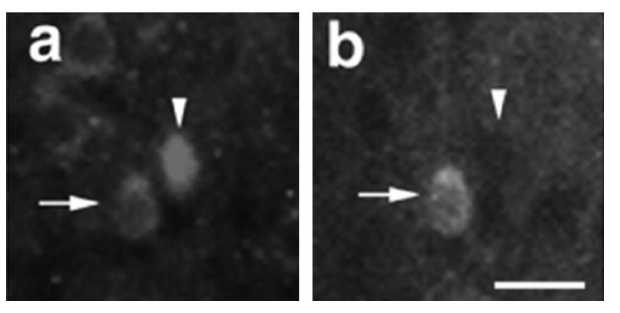

Figure 3. Calbindin-containing cells in lateral septum are TrkB-positive. $a$, A fluorescence micrograph showing immunoreactivity for TrkB (arrow) on a lateral septal neuron that also stains for calbindin $(b)$. Note that all calbindin-positive cells are also TrkB-positive (arrows), but not all TrkBcontaining cells contain calbindin (arrowhead).

peptide fragment in mature BDNF, the same blot showed a slight increase in BDNF levels, consistent with the overexpression of $\mathrm{BDNF}$ in noradrenergic neurons.

We also examined calbindin levels in the septa of DBH-BDNF transgenic mice, knowing that the LC, which overexpresses BDNF in these animals (Fawcett et al., 1998), projects through the MFB into the septal region (Jakab and Leranth, 1995). Western blot replicas from the septa of two separate control and transgenic animals show increased calbindin expression (Fig. 4b), suggesting that overexpression of BDNF in specific noradrenergic neurons increases calbindin expression in target tissues.

In a second series of experiments, we used 6-OHDA to lesion ascending catecholaminergic axons coursing through the MFB. We reasoned that if BDNF transported anterogradely in catecholaminergic axons is important for the regulation of calbindin in postsynaptic neurons, then removal of BDNF-containing axons in the lateral septum would result in a decrease in calbindin expression. Unilateral injections of 6-OHDA, which destroys catecholaminergic axons and neurons (Ungerstedt, 1968), were made into the MFB of adult rats. Four to six weeks after
6-OHDA injection, individual septa were dissected and separated into lesioned and unlesioned sides. Equal amounts of lysate were loaded from lesioned and nonlesioned septa, as determined by tubulin measurements (Fig. 4C, top panel). Figure $4 C$ (middle panel) shows the almost complete absence of $\mathrm{TH}$ immunoreactivity in the lesioned septa, confirming that the lesion successfully disrupted afferent catecholaminergic input to the septum. Figure $4 C$ (bottom panel) shows a significant reduction, but not complete loss, of calbindin expression on the lesioned side compared with the control side (Fig. 4C, bottom panel). Densitometry scans, normalizing calbindin levels to tubulin, were done for both the lesioned and nonlesioned sides from three separate animals. Data revealed a $36 \pm 6 \%(p<0.01 ; n=3)$ reduction in calbindin after 6-OHDA lesion (Fig. 4D). Thus, removal of catecholaminergic axons leads to a loss in calbindin expression in the septum.

To determine whether loss of $\mathrm{TH}$ fibers also lead to a loss in BDNF immunoreactivity in the lateral septum, we turned to double immunocytochemistry. Reductions in staining for $\mathrm{TH}$ (Fig. 4E, top panels) and BDNF (Fig. 4E, bottom panels) were evident on the lesioned versus control sides. Not all BDNF immunoreactivity disappeared on the lesioned side, suggesting that there are other sources of BDNF protein into the lateral septum that were not affected by the 6-OHDA lesion. Thus, loss of catecholaminergic afferent nerve fibers lead to a loss of BDNF immunoreactivity in the lateral septum.

\section{BDNF promotes calbindin synthesis in cultured septal neurons}

Although our in vivo results suggest that BDNF in presynaptic neurons regulates calbindin levels in postsynaptic cells, we cannot rule out an indirect effect of BDNF nor the possibility that catecholamines themselves influence calbindin levels. To determine whether BDNF directly affects calbindin levels in septal neurons, we established cultures of septal neurons from E16-E17 rat embryos and incubated them in medium with or without 
A

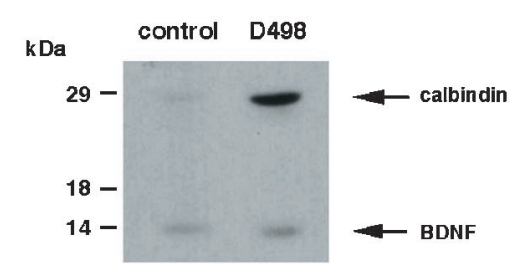

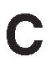

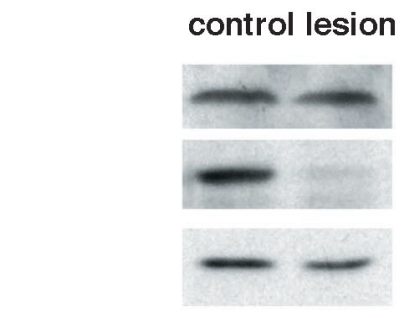

Tubulin

$\mathrm{TH}$

Calbindin
Figure 4. Changes in brain levels of calbindin, tyrosine hydroxylase, and BDNF. $A$, A Western blot replica showing increased levels of calbindin and BDNF in lysates of whole brain from control and transgenic mice overexpressing BDNF in catecholaminergic neurons under the control of a $1.6 \mathrm{~kb}$ fragment of the $\mathrm{DBH}$ promoter. Fifty micrograms of protein were loaded in each lane. $B$, Western blot replica showing the level of calbindin expression in the septa dissected from two control and two $\mathrm{DBH}-$ BDNF transgenic mice. Extracts from separate mice were tested in each lane. $C$ Western blot replicas measuring levels of tubulin, $\mathrm{TH}$, and calbindin in septa taken from the control and lesioned sides 4 weeks after unilateral injections of 6-OHDA to disrupt the medial forebrain bundle. Samples from one representative animal are shown. $D$, Densitometry of the Western blot replicas, as shown in $C$. Calbindin levels were normalized to tubulin from three separate animals. Asterisks indicate statistical significance as determined by paired $t$ tests. $E$, Immunofluorescent micrographs showing TH (top panels) and BDNF (bottom panels) in the lateral septum without lesion (Control, left panels) and after treatment with 6-OHDA (Lesion, right panel). Note the decrease in TH and BDNF immunoreactivity on the lesioned side compared with control side. $L S$, Lateral septum; $L V$, lateral ventricle. Scale bar, $50 \mu \mathrm{m}$.
B

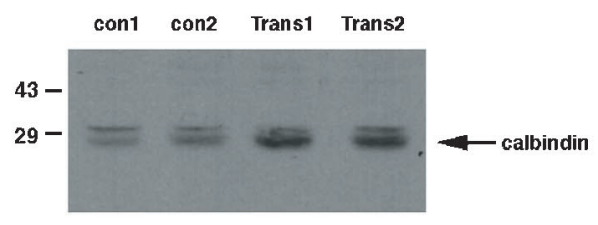

E
BDNF. Two days after plating, $50 \mathrm{ng} / \mathrm{ml}$ BDNF was added and replenished every other day, for up to $5 \mathrm{~d}$. Western blot analysis of cell lysates (Fig. $5 A$ ) revealed significant increases in calbindin expression in cultures treated with BDNF. This effect was dosedependent (data not shown) within the ranges reported for the effects of BDNF on cultured hippocampal neurons (Ip et al., 1993).

To characterize more fully the increase in calbindin, we turned to immunocytochemistry, which revealed that BDNF had a profound effect on the number and structure of calbindin-containing neurons (Fig. 5B,C). Cultures treated with BDNF contained more calbindin-positive neurons (treated $8.3 \pm 0.43$ vs control $3.5 \pm 0.4$ per field of view at $40 \times ; p<0.001)$ than cultures not receiving $\mathrm{BDNF}$ (Fig. $5 B$, C, left panels). BDNF-treated neurons also appeared to be larger than neurons grown without BDNF, and they elaborated from their cell bodies significantly more primary neurites than untreated cells (treated $4.5 \pm 0.35$ vs control $2.8 \pm 0.18$ per field of view at $40 \times ; p<0.001$ ) (Fig. $6 c$, right panel). However, BDNF had no effect on the total number of cells in the culture, as determined with Hoechst 33258 staining (data not shown). Thus, exogenous BDNF increases the expression of calbindin and neurite arborization from cultured septal neurons.
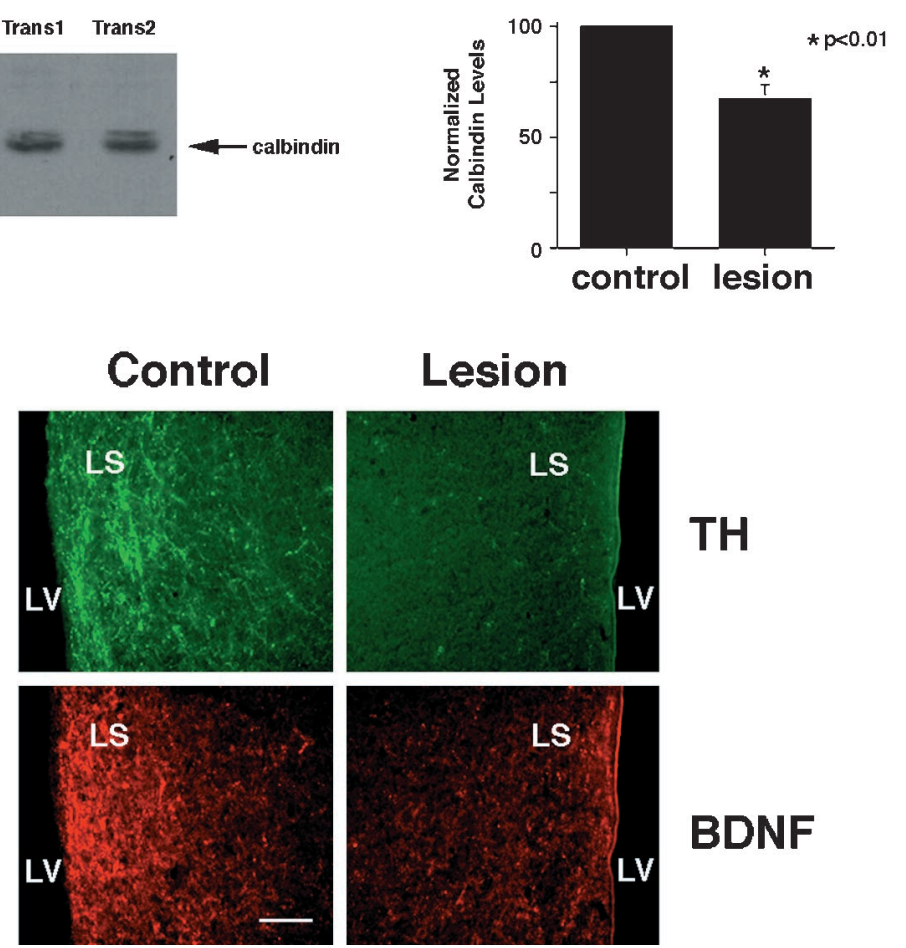

Lesion

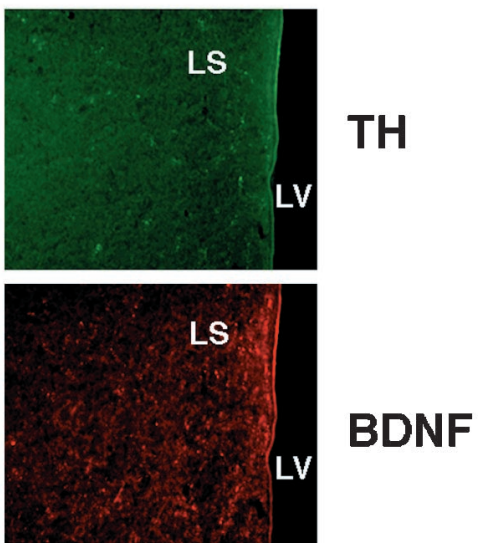

To determine whether the effects of BDNF on calbindincontaining cells arose from BDNF acting through the TrkB receptor, we repeated the experiment in cultures treated with the tyrosine kinase inhibitor K252a (200 nM). Under these conditions, BDNF failed to increase either the number of calbindincontaining neurons (control $3.5 \pm 0.4$ vs BDNF/K252a $2.5 \pm 0.35$ per field of view at $40 \times ; p>0.05$ ) (Fig. $6 c$, left panel) or the number of primary neurites emanating from cell bodies (control $2.8 \pm 0.18$ vs BDNF/K252a $2.5 \pm 0.8$ per field of view at $40 \times ; p>$ $0.05)$ (Fig. 5C, right panel). Thus, BDNF appears to be exerting its effects on calbindin-positive neurons through tyrosine kinase activation, presumably TrkB.

To determine whether BDNF increased the number of calbindin-containing cells in these cultures by promoting cell division, we double-labeled cells with BrdU and calbindin (Fig. $6 a-d)$. Results showed that $5.8 \pm 0.2 \%$ of calbindin-containing cells were positive for BrdU in the presence of BDNF compared with $7.0 \pm 0.3 \%$ in the absence of BDNF, differences that were not significant (Fig. 6e, left panel). In these cultures, there were clear examples of calbindin-containing cells (Fig. 6a, arrows) that are negative for $\operatorname{BrdU}(b)$, although occasionally calbindin- 
A

$-B D N F+B D N F$

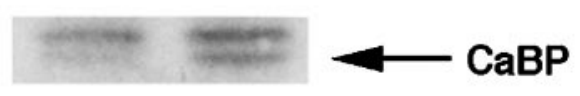

B

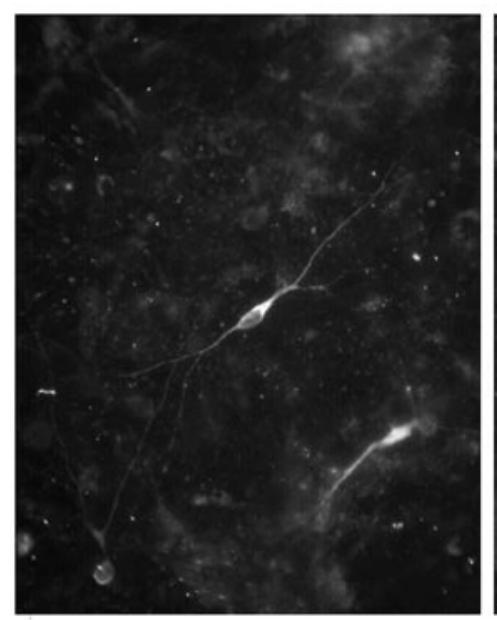

-BDNF
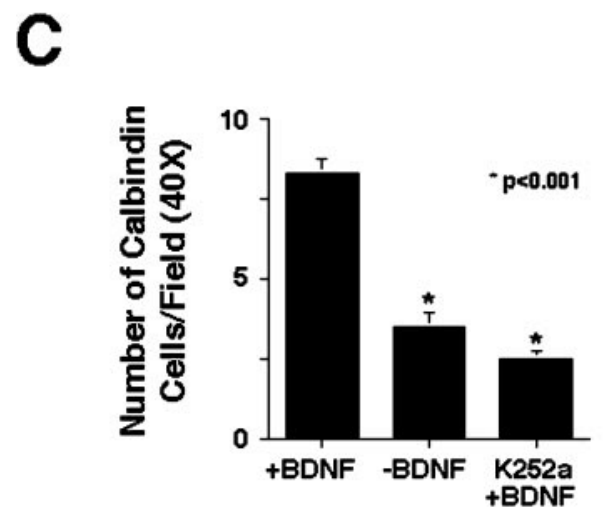

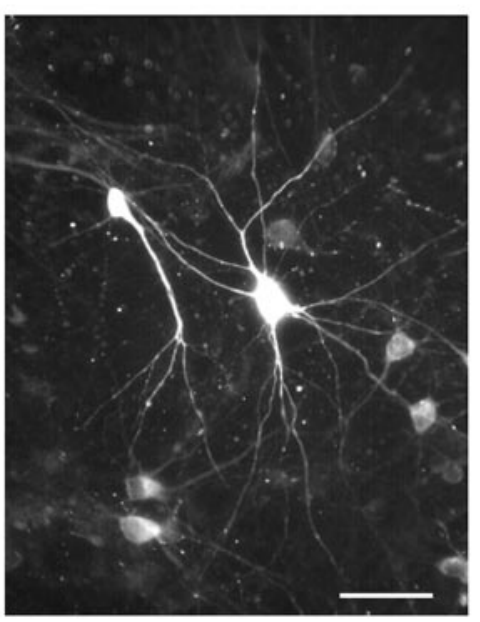

+BDNF
Figure 5. The effects of BDNF on calbindin levels in cultured septal neurons. $A$, Septal neurons from E16 rats cultured for $5 \mathrm{~d}$ with 50 $\mathrm{ng} / \mathrm{ml}$ BDNF produce more calbindin $(\mathrm{CaBP})$ than untreated cells $(-B D N F)$. Fifty micrograms of total protein were loaded in each lane. $B$, Fluorescent micrograph of calbindin immunoreactivity in cells from septum cultured for $5 \mathrm{~d}$ in the presence or absence of BDNF (50 $\mathrm{ng} / \mathrm{ml})$. C, Left panel, BDNF treatment led to an increase in the number of calbindin-positive cells in culture, scored per field of view at $40 \times$. Cultures treated with K252a and BDNF showed no increases relative to controls. Right panel, BDNF treatment increased the number of primary neurites emanating from the cell body of calbindin-containing neurons, an effect that was ablated by K252a. Asterisks indicate statistical significance as determined by Student's $t$ tests. Scale bar, $25 \mu \mathrm{m}$. containing cells labeled with BrdU are evident ( $a-d$, arrowhead). Cells negative for both calbindin and $\operatorname{BrdU}(a-d$, asterisks) were also evident. Thus, BDNF does not induce proliferation of calbindin-positive neurons.

To determine whether BDNF increased the number of calbindin-containing cells by reducing the rate of cell death, we used terminal deoxynucleotidyl transferase-mediated biotinylated UTP nick end labeling (TUNEL) to measure the number of cells undergoing apoptotic death after $2 \mathrm{~d}$ of BDNF treatment. Although very few cells were TUNEL-positive (data not shown), there were no differences in the number of cells undergoing apoptosis in treated $(1.54 \pm 0.23)$ versus untreated $(1.45 \pm .014)$ cultures (per field of view at $20 \times ; p>0.05$ ). Together, these data suggest that BDNF directly alters the structure and phenotype of postmitotic calbindin-containing neurons rather than promote the proliferation or survival of calbindin-producing neuronal precursors.

\section{DISCUSSION}

Data in this study show that BDNF produced in catecholaminergic brainstem neurons is anterogradely transported within the MFB and modulates the expression of calbindin in the lateral septum. BDNF-containing nerve terminals synapse on calbindincontaining neurons that produce TrkB, the high-affinity receptor for BDNF. BDNF treatment increased calbindin expression and neurite outgrowth in cultured postmitotic embryonic septal neurons but had no effect on rate of cell division or cell death. Therefore, an important function of the BDNF transported from nerve centers outside the septum may be to regulate the chemical and structural maturation of calbindin-containing septal neurons.

\section{Lesions of the MFB}

BDNF is known to be transported anterogradely in noradrenergic neurons (Fawcett et al., 1997). Data in this study extend that finding by showing that BDNF is anterogradely transported into lateral septum within catecholaminergic fibers of the MFB. Two 

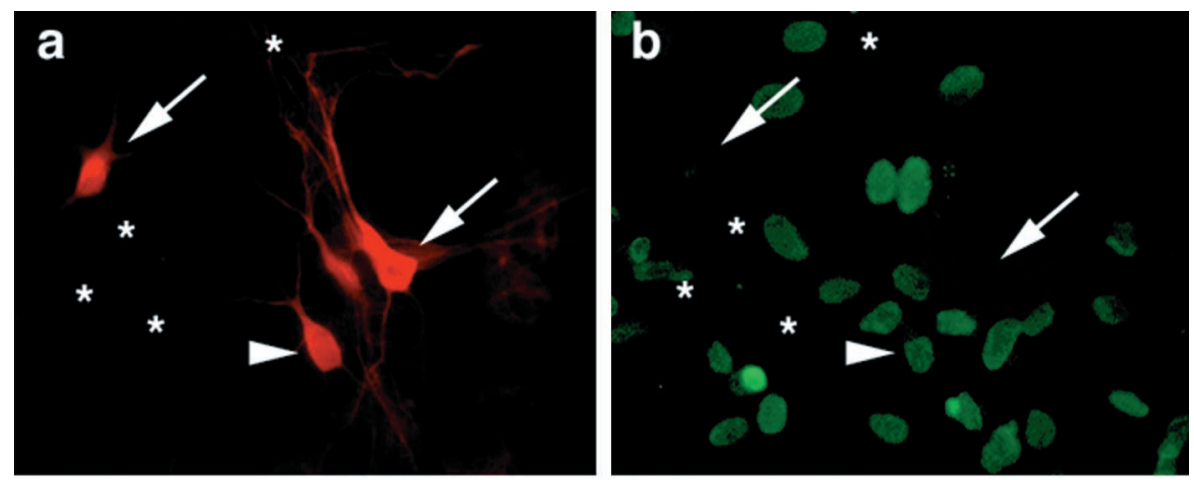

Figure 6. BDNF does not promote cell division or inhibit cell death. Staining of septal cultures for calbindin $(a), \operatorname{BrdU}(b)$, Hoechst (c), and all three markers $(d)$. A small population of calbindin-positive cells ( $a$, arrowhead) were also positive for $\mathrm{BrdU}$ ( $b$ and $d$, arrowheads); however, the majority of calbindin-positive cells ( $a$, arrows) were BrdU-negative ( $b$ and $d$, arrows), suggesting little if any proliferation in calbindinpositive cells. Some cells were calbindin-negative (a, asterisks) and BrdU-negative ( $b$, asterisks) but Hoechst 33258-positive ( $c$ and $d$, asterisks). e, Left panel, There was no significant difference in the number of calbindin-positive cells relative to BrdU-positive cells in the presence or absence of BDNF. Similarly, BDNF had no effect on the number of TUNEL-positive cells (right panel). We found no examples of calbindin-positive cells that were also TUNEL-positive (data not shown). Scale bar, $25 \mu \mathrm{m}$.
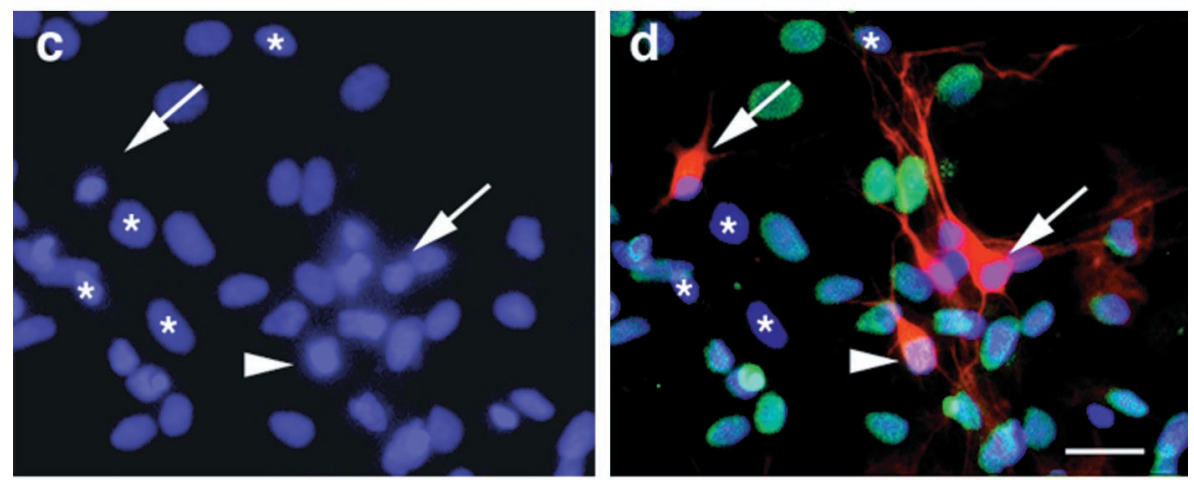

lines of evidence support this idea. First, BDNF and TH colocalize in axons and terminals of fibers within the lateral septum, supporting the notion that the two molecules are anterogradely transported in catecholaminergic neurons. Second, lesion experiments in which the MFB was damaged unilaterally with 6-OHDA resulted in a significant decrease in TH and BDNF immunoreactivity within the ipsilateral lateral septum compared with the unlesioned control side (Figs. 2, 3). Catecholaminergic fibers in the MFB that transport BDNF likely arise from the VTA, SNPC, and supermammilary nucleus. All these nerve centers send dopaminergic axons to the lateral septum via the MFB (Swanson, 1982; Jakab and Leranth, 1995) and synthesize BDNF mRNA (Conner et al., 1997) (see also Seroogy et al., 1994; Venero et al., 1994). Fibers from these midbrain nuclei also form pericellular basket endings around calbindin-positive neurons in the lateral septum (Seifert et al., 1998), which is the same appearance we observed for BDNF-containing nerve fibers in lateral septum (Figs. 1c,d,2). Consistent with these results are data from others showing that intraventricular injection of colchicine, a blocker of axonal transport, increases BDNF immunoreactivity in the SNPC, probably as a result of protein accumulation (Altar et
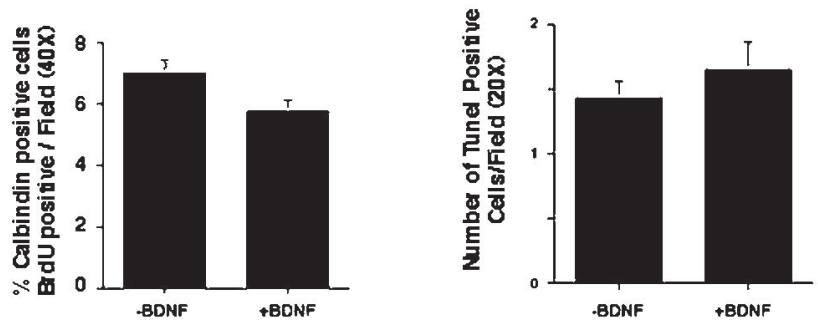

al., 1997). The LC is probably not a major source of BDNF for the lateral septum because axons arising from the LC pass through the lateral septum without forming pericellular basket endings (Jakab and Leranth, 1995). However, we cannot rule out the possibility that collateral branches from ascending LC fibers contact calbindin-containing neurons. Indeed, in transgenic mice that overexpress BDNF in noradrenergic neurons, levels of calbindin are increased in the septum.

Evidence that BDNF is anterogradely transported in the MFB is consistent with the work of others who have monitored the anterograde transport of BDNF in axons of both the CNS and peripheral nervous system (for review, see Altar and DiStephano, 1998). BDNF has also been detected in a vesicular fraction of brain synaptosomes (Fawcett et, 1997), consistent with the localization of BDNF in large-dense core vesicles (Michael et al., 1997). Furthermore, BDNF is sorted into the regulated secretory pathway of neurons, which is consistent with it being released by activity-dependent mechanisms from presynaptic terminals (Aloyz et al., 1999; Mowla et al., 1999).

Catecholaminergic fibers within the MFB are not the only source of BDNF in lateral septum, because BDNF immunoreac- 
tivity remained in lateral septum after MFB lesions (Fig. 4E). Neurons containing mRNA coding for BDNF are present in the medial septum, as well as in hippocampus (Castren et al., 1995). These neurons send fibers and perhaps deliver BDNF to the more lateral regions of the septum. Further work is necessary to clearly define noncatecholaminergic sources of BDNF to this region.

Within the lateral septum, BDNF and $\mathrm{TH}$ colocalize within nerve fibers (Fig. 1), but not all TH-containing fibers contain BDNF. This suggests that different populations of catecholaminergic neurons innervate the lateral septum. As well, BDNFcontaining fibers terminate on a population of calbindincontaining cells that also contain TrkB (Fig. 3). Not all TrkB containing-cells contain calbindin, but all calbindin-containing cells contain TrkB (Fig. 3), again suggesting the presence of multiple populations of cells in septum.

\section{BDNF regulation of calbindin-containing neurons}

BDNF-containing catecholaminergic nerve fibers surround TrkBand calbindin-containing neurons, suggesting that BDNF may play a role in regulating the function of calbindin-containing postsynaptic neurons. This idea has been confirmed both in vivo and in vitro. Calbindin levels in septum (Fig. 4B), as well as in whole brain (Fig. 4A), were elevated in transgenic mice that overexpress BDNF in catecholaminergic neurons under the control of the dopamine- $\beta$-hydroxylase promoter. Conversely, others have shown that calbindin levels are reduced in brains from BDNF null mice (Jones et al., 1994). Furthermore, in our studies, BDNF induced significant changes in septal neurons from E17 embryos grown for $5 \mathrm{~d}$ in culture. BDNF increased the total amount of calbindin expressed by cultures of septal neurons, presumably by increasing the number and arborization of cells that express calbindin (Fig. 5B,C). These effects were mediated by the TrkB receptor because they did not occur in cells exposed to the tyrosine kinase inhibitor K252a. BDNF did not effect the number of cells staining with BrdU and had no effect on the number of TUNEL-positive neurons. Therefore, BDNF is promoting the chemical and structural maturation of postmitotic neurons without effecting the rate of cell death. These results are consistent with whole animal studies showing that loss of trophic support induced by deafferentation (Linden, 1994) reduces the size of target neurons and atrophy of dendrites without promoting cell death (Tierney et al., 1997) (for review, see Sherrard and Bower, 1998).

Our data agree with the results of others who have shown that BDNF increases calbindin levels in cultures of postmitotic neurons from hippocampus (Ip et al., 1993), striatum (Mizuno et al., 1994; Ventigmiglia et al., 1995), cerebral cortex (Pappas and Parnavelas, 1997), and cerebellum (Larkfors et al., 1996). BDNF can also alter the morphology of neurons in the brain (Marty et al., 1996; Inoue and Sanes, 1997; Shimada et al., 1998), including increasing the density of synaptic terminals (Causing et al., 1997), probably by promoting dendritic (McAllister et al., 1995, 1996) and axonal arborization (Cohen-Corey and Fraser, 1995). Conversely, dendrite arborization in Purkinje cells is reduced in BDNF null mice (Schwartz et al., 1997), and TrkB null mutant mice show reduced synaptic contacts, as well as a selective loss of synaptic vesicle proteins (Martinez et al., 1998). Together, all of these data support the idea that BDNF plays an essential role in the developmental maturation of neurons in the mammalian CNS.

The discovery that lesions of the MFB in adult rats leads reduced $\mathrm{BDNF}$ and calbindin-expression in the lateral septum could be of use experimentally for several reasons. First, this may be an ideal system for learning more about the physiology of
BDNF in normal brain. Although we know much about where BDNF is made in brain and increasingly about its effects in promoting nerve cell function and synaptic plasticity, few studies have described an intact neural network in which the production, transport, and effects of BDNF on postsynaptic neurons can be analyzed. Previous studies have shown that NGF produced in hippocampus is retrogradely transported within basal forebrain cholinergic neurons, which require the protein for survival. This system has been exploited for studying the production, transport, and effects of NGF in the CNS (Hefti, 1986; Hefti et al., 1989; Fischer et al., 1991; Van der Zee et al., 1992; Chen et al., 1997). In a similar way, studying the production of BDNF in brainstem neurons, its anterograde transport in the MFB, and its effects within the lateral septum of adult rodents could be a useful model for better understanding the physiological role of BDNF in brain.

Furthermore, this same system could be useful for understanding cognitive changes associated with loss of monoaminergic neurons in normal aging and neurodegenerative disease (Jellinger, 1996). Progressive loss of brainstem dopaminergic neurons occurs in normal aging (Kish et al., 1992). Mesolimbic dopaminergic and locus ceruleus noradrenergic neurons when lost in Parkinson's disease result in cognitive impairment (Kopin and Markey, 1988). Understanding how BDNF in monoaminergic systems regulates calbindin-containing neurons in the adult lateral septum may provide useful insight into mechanisms of cognitive impairment in neurodegenerative disease. Interestingly, BDNF protein is reduced in septum of aged rats (Katoh-Semba et al., 1998). Furthermore, calbindin expression is reduced in the septal complex of patients with Parkinson's disease (Chan-Palay et al., 1993). We demonstrate that loss of catecholaminergic input to the lateral septum is associated with decreased calbindinexpression as a result of reduced anterograde transport of BDNF. Such a system may serve as useful model for understanding cognitive impairment associated with monoaminergic dysfunction.

\section{REFERENCES}

Aloyz RA, Fawcett, JP, Kaplan DR, Murphy RA, Miller FD (1999) Activity-dependent activation of TrkB neurotrophin receptors in the adult CNS. Learn Mem 6:216-231.

Altar CA, DiStephano PS (1998) Neurotrophin trafficking by anterograde transport. Trends Neurosci 21:433-437.

Altar CA, Cai N, Bliven T, Juhasz M, Conner JM, Acheson AL, Lindsay RM, Wiegand SJ (1997) Anterograde transport of brain-derived neurotrophic factor and its role in the brain. Nature 389:856-860.

Armstrong DM, Pickel VM, Joh TH, Reis DJ, Miller RJ (1981) Immunocytochemical localization of catecholamine synthesizing enzymes and neuropeptides in area postrema and medial nucleus tractus solitarius of rat brain. J Comp Neurol 196:505-517.

Baimbridge KG, Celio MR, Rogers JH (1992) Calcium binding proteins in the nervous system. Trends Neurosci 15:303-308.

Castren E, Thoenen H, Lindholm D (1995) Brain-derived neurotrophic factor messenger RNA is expressed in the septum, hypothalamus and in adrenergic brain stem nuclei of adult rat brain and is increased by osmotic stimulation in the paraventricular nucleus. Neuroscience 64:71-80.

Causing CG, Gloster A, Aloyz R, Bamji SX, Chang E, Fawcett J, Kuchel G, Miller FD (1997) Synaptic innervation density is regulated by neuron-derived BDNF. Neuron 18:257-267.

Chan-Palay V, Hochli M, Savaskan E, Hungerecker G (1993) Calbindin $\mathrm{D}-28 \mathrm{k}$ and monoamine oxidase A immunoreactive neurons in the nucleus basalis of Meynert in senile dementia of the Alzheimer type and Parkinson's disease. Dementia 4:1-15.

Chen KS, Nishimura MC, Armanini MP, Crowley C, Spencer SD, Phillips HS (1997) Disruption of a single allele of the nerve growth factor gene results in atrophy of basal forebrain cholinergic neurons and memory deficits. J Neurosci 17:7288-7296.

Cohen-Cory S, Fraser SE (1995) Effects of brain-derived neurotrophic factor on optic axon branching and remodelling in vivo. Nature 378:192-196. 
Conner JM, Lauterborn JC, Yan Q, Gall CM, Varon S (1997) Distribution of brain-derived neurotrophic factor (BDNF) protein and mRNA in the normal adult rat CNS: evidence for anterograde axonal transport. J Neurosci 17:2295-2313.

Cortes R, Ceccatelli S, Schalling M, Hokfelt T (1990) Differential effects of intracerebroventricular colchicine administration on the expression of mRNAs for neuropeptides and neurotransmitter enzymes, with special emphasis on galanin: an in situ hybridization study. Synapse 6:369-391.

Fawcett JP, Aloyz R, McLean JH, Pareek S, Miller FD, McPherson PS, Murphy RA (1997) Detection of brain-derived neurotrophic factor in a vesicular fraction of brain synaptosomes. J Biol Chem 272:8837-8840.

Fawcett JP, Bamji SX, Causing CG, Aloyz R, Ase AR, Reader TA, McLean JH, Miller FD (1998) Functional evidence that BDNF is an anterograde neuronal trophic factor in the CNS. J Neurosci 18:2808-2821.

Fischer W, Nilsson OG, Bjorklund A (1991) In vivo acetylcholine release as measured by microdialysis is unaltered in the hippocampus of cognitively impaired aged rats with degenerative changes in the basal forebrain. Brain Res 556:44-52.

Fryer RH, Kaplan DR, Feinstein SC, Radeke MJ, Grayson DR, Kromer LF (1996) Developmental and mature expression of full-length and truncated TrkB receptors in the rat forebrain. J Comp Neurol 374:21-40.

Goodman LJ, Valverde J, Lim F, Geschwind MD, Federoff HJ, Geller AI, Hefti F (1996) Regulated release and polarized localization of brain-derived neurotrophic factor in hippocampal neurons. Mol Cell Neurosci 7:222-238.

Hefti F (1986) Nerve growth factor promotes survival of septal cholinergic neurons after fimbrial transections. J Neurosci 6:2155-2162.

Hefti F, Hartikka J, Knusel B (1989) Function of neurotrophic factors in the adult and aging brain and their possible use in the treatment of neurodegenerative diseases. Neurobiol Aging 10:515-533.

Inoue A, Sanes JR (1997) Lamina-specific connectivity in the brain: regulation by $\mathrm{N}$-cadherin, neurotrophins, and glycoconjugates. Science 276:1428-1431.

Ip N, Li Y, Yancopoulos G, Lindsay RM (1993) Cultured hippocampal neurons show responses to BDNF, NT-3, NT-4 but not NGF. J Neurosci 13:3394-3405.

Jaffard R, Vouimba RM, Marighetto A, Garcia R (1996) Long-term potentiation and long-term depression in the lateral septum in spatial working and reference memory. J Physiol (Paris) 90:339-341.

Jakab RL, Leranth C (1990) Catecholaminergic, GABAergic, and hippocamposeptal innervation of GABAergic "somatospiny" neurons in the rat lateral septal area. J Comp Neurol 302:305-321.

Jakab RL, Leranth C (1995) Septum. In: Rat nervous system (Paxinos G, ed), pp 405-442. New York: Academic.

Jellinger KA (1996) Structural basis of dementia in neurodegenerative disorders. J Neural Transm 47:1-29.

Jones, KR, Farinas I, Backus C, Reichardt LF (1994) Target disruption of BDNF gene perturbs brain and sensory neuron development but not motor neuron development. Cell 76:989-999.

Katoh-Semba R, Semba R, Takeuchi IK, Kato K (1998) Age-related changes in levels of brain-derived neurotrophic factor in selected brain regions of rats, normal mice and senescence-accelerated mice: a comparison to those of nerve growth factor and neurotrophin-3. Neurosci Res 31:227-234.

Kish SJ, Shannak K, Rajput A, Deck JH, Hornykiewicz O (1992) Aging produces a specific pattern of striatal dopamine loss: implications for the etiology of idiopathic Parkinson's disease. J Neurochem 58:642-648.

Kopin and IJ, Markey SP (1988) MPTP toxicity: implications for research in Parkinson's disease. Annu Rev Neurosci 11:81-96.

Larkfors L, Lindsay RM, Alderson RF (1996) Characterization of the responses of Purkinje cells to neurotrophin treatment. J Neurochem 66:1362-1373.

Linden R (1994) The survival of developing neurons: a review of afferent control. Neuroscience 4:671-682.

Lindvall O (1975) Mesencephalic dopaminergic afferents to the lateral septal nucleus of the rat. Brain Res 87:89-95.

Martinez A, Alcantara S, Borrell V, Del Rio JA, Blasi J, Otal R, Campos N, Boronat A, Barbacid M, Silos-Santiago I, Soriano E (1998) TrkB and TrkC signaling are required for maturation and synaptogenesis of hippocampal connections. J Neurosci 18:7336-7350.

Marty S, Carroll P, Cellerino A, Castren E, Staiger V, Thoenen H, Lindholm D (1996) Brain-derived neurotrophic factor promotes the differentiation of various hippocampal nonpyramidal neurons, including Cajal-Retzius cells, in organotypic slice cultures. J Neurosci 16:675-687.
Mazzoni IE, Kenigsberg RL (1991) Thrombin indirectly affects cholinergic cell expression in primary septal cell cultures in a manner distinct from nerve growth factor. Neuroscience 45:195-204.

McAllister AK, Lo DC, Katz LC (1995) Neurotrophins regulate dendritic growth in developing visual cortex. Neuron 15:791-803.

McAllister AK, Katz LC, Lo DC (1996) Neurotrophin regulation of cortical dendritic growth requires activity. Neuron 17:1057-1064.

McAllister AK, Katz LC, Lo DC (1999) Neurotrophins and synaptic plasticity. Annu Rev Neurosci 22:295-318.

Michael GJ, Averill S, Nitkunan A, Rattray M, Bennett DL, Yan Q, Priestley JV (1997) Nerve growth factor treatment increases brainderived neurotrophic factor selectively in TrkA-expressing dorsal root ganglion cells and in their central terminations within the spinal cord. J Neurosci 17:8476-8490.

Mizuno K, Carnahan J, Nawa H (1994) Brain-derived neurotrophic factor promotes differentiation of striatal GABAergic neurons. Dev Biol 165:243-256.

Mowla SJ, Pareek S, Farhadi H, Petrecca K, Fawcett JP, Seidah NG, Morris SJ, Sossin WS, Murphy RA (1999) Differential sorting of NGF and BDNF in hippocampal neurons J Neurosci 19:2069-2080.

Pappas IS, Parnavelas JG (1997) Neurotrophins and basic fibroblast growth factor induce the differentiation of calbindin-containing neurons in the cerebral cortex. Exp Neurol 144:302-314.

Schwartz PM, Borghesani PR, Levy RL, Pomeroy SL, Segal RA (1997) Abnormal cerebellar development and foliation in BDNF $-/-$ mice reveals a role for neurotrophins in CNS patterning. Neuron 19:269-281.

Seifert U, Hartig W, Grosche J, Bruckner G, Riedel A, Brauer K (1998) Axonal expression sites of tyrosine hydroxylase, calretinin, and calbindin-immunoreactivity in striato-pallidal and septal nuclei $f$ the rat brain: a double-immunolabelling study. Brain Res 795:227-246.

Seroogy KB, Gall CM (1993) Expression of neurotrophins by midbrain dopaminergic neurons. Exp Neurol 124:119-128.

Seroogy KB, Lundgren KH, Tran TM, Guthrie KM, Isackson PJ, Gall CM (1994) Dopaminergic neurons in rat ventral midbrain express brain-derived neurotrophic factor and neurotrophin-3 mRNAs. J Comp Neurol 342:321-324.

Sherrard RM, Bower A (1998) Role of afferents in the development and cell survival of the vertebrate nervous system. Clin Exp Pharmacol Physiol 25:487-495.

Shimada A, Mason CA, Morrison ME (1998) TrkB signaling modulates spine density and morphology independent of dendrite structure in cultured neonatal Purkinje cells. J Neurosci 18:8559-8570.

Snider WD (1994) Functions of the neurotrophins during nervous system development: what the knockouts are teaching us. Cell 77:627-638.

Swanson LW (1982) The projections of the ventral tegmental area and adjacent regions: a combined fluorescent retrograde tracer and immunofluorescent study in the rat. Brain Res Bull 9:321-353.

Thoenen H (1995) Neurotrophins and neuronal plasticity. Science 270:593-598.

Tierney TS, Russell FA, Moore DR (1997) Susceptibility of developing cochlear nucleus neurons to deafferentation-induced death abruptly ends just before the onset of hearing. J Comp Neurol 378:295-306.

Ungerstedt U (1968) 6-Hydroxy-dopamine induced degeneration of central monoamine neurons. Eur J Pharmacol 5:107-110.

Urban IJ, Ontskul A, Croiset G, Cheng Y, de Wied D (1995) A longlasting increase and decrease in synaptic excitability in the rat lateral septum are associated with high and low shuttle box performance, respectively. Behav Brain Res 68:173-183.

Van der Zee CE, Fawcett J, Diamond J (1992) Antibody to NGF inhibits collateral sprouting of septohippocampal fibers following entorhinal cortex lesion in adult rats. J Comp Neurol 326:91-100.

Ventimiglia R, Mather PE, Jones BE, Lindsay RM (1995) The neurotrophins BDNF, NT-3 and NT-4/5 promote survival and morphological and biochemical differentiation of striatal neurons in vitro. Eur J Neurosci 7:213-222.

Venero JL, Beck KD, Hefti F (1994) 6-Hydroxydopamine lesions reduce BDNF mRNA levels in adult rat brain substantia nigra. NeuroReport $5: 429-432$

von Bartheld CS, Byers MR, Williams R, Bothwell M (1996) Anterograde transport of neurotrophins and axodendritic transfer in the developing visual system. Nature 379:830-833.

Yan Q, Rosenfeld RD, Metheson CR, Hawkins N, Lopez OT, Bennett L, Welcher AA (1997) Expression of brain derived neurotrophic factor protein in the adult rat central nervous system Neuroscience 78:431-448. 